\title{
Unveiling Potential Active Constituents and Pharmacological Mechanisms of Pudilanxiaoyan Oral Liquid for Anti-Coronavirus Pneumonia Using Network Pharmacology
}

\author{
Ying-Peng Tong ${ }^{1}$ Xiao-Fei Shen ${ }^{2}$ \\ Zi-Ping Zhu ${ }^{1}$ Jian-Xin Wang ${ }^{1,4,5^{*}}$ \\ ${ }^{1}$ Institute of Natural Medicine and Health Product, School of Advanced \\ Study, Taizhou University, Taizhou, People's Republic of China \\ ${ }^{2}$ TCM Regulating Metabolic Diseases Key Laboratory of Sichuan \\ Province, Hospital of Chengdu University of Traditional Chinese \\ Medicine, Chengdu, People's Republic of China \\ 3 Jiangsu Key Laboratory of Chinese Medicine and Characteristic \\ Preparations for Paediatrics, Jumpcan Pharmaceutical Co., Ltd., \\ Taizhou, People's Republic of China \\ ${ }^{4}$ Department of Pharmaceutics, School of Pharmacy, Fudan \\ University \& Key Laboratory of Smart Drug Delivery, Ministry of \\ Education, Shanghai, People's Republic of China \\ ${ }^{5}$ Institute of Integrative Medicine, Fudan University, Shanghai, \\ People's Republic of China
}

Chao Li ${ }^{3}$ Qi Zhou ${ }^{1}$ Chun-Xiao Jiang ${ }^{1} \quad \mathrm{Na} \mathrm{Li}^{1} \quad$ Zhen-Da Xie $^{1}$

Pharmaceut Fronts 2021;3:e65-e76.

\begin{abstract}
Address for correspondence Jian-Xin Wang, PhD, Department of Pharmaceutics, School of Pharmacy, Key Laboratory of Smart Drug Delivery (Fudan University), Ministry of Education, 826 Zhangheng Road, Pudong New District, Shanghai 201203, People's Republic of China (e-mail: jxwang@fudan.edu.cn).
\end{abstract}

\begin{abstract}
Keywords

- pudilanxiaoyan oral liquid

- COVID-19

- active ingredients

- network pharmacology

- NF-kB signaling pathway

The outbreak of novel coronavirus pneumonia (COVID-19), defined as a worldwide pandemic, has been a public health emergency of international concern. Pudilanxiaoyan oral liquid (PDL), an effective drug of Traditional Chinese Medicine (TCM), is considered to be an effective and alternative means for clinical prevention of COVID-19. The purpose of this study was to identify potential active constituents of PDL, and explore its underlying anti-COVID-19 mechanism using network pharmacology. Integration of target prediction (SwissTargetPrediction and STITCH database) was used to elucidate the active components of PDL. Protein-protein interaction network analyses, gene ontology, Kyoto Encyclopedia of Genes and Genomes pathway enrichment analyses, network construction, and molecular docking were applied to analyze the prospective mechanisms of the predicted target genes. Our results showed that the key active ingredients in PDL were luteolin, apigenin, esculetin, chrysin, baicalein, oroxylin A, baicalin, wogonin, cymaroside, and gallic acid. A majority of the predicted targets were mainly involved in the pathways related to viral infection, lung injury, and inflammatory responses. An in vitro study further inferred that inhibiting the activity of nuclear factor (NF)-кB signaling pathway was a key mechanism by which PDL exerted anti-COVID-19 effects. This study not only provides chemical basis and pharmacology of PDL but also the rationale for strategies to exploring future TCM for COVID-19 therapy.
\end{abstract}

received

May 9, 2021

accepted

June 22, 2021
DOI https://doi.org/

$10.1055 / \mathrm{s}-0041-1735147$. ISSN 2628-5088. (c) 2021. The Author(s).

This is an open access article published by Thieme under the terms of the Creative Commons Attribution License, permitting unrestricted use, distribution, and reproduction so long as the original work is properly cited. (https://creativecommons.org/licenses/by/4.0/)

Georg Thieme Verlag KG, Rüdigerstraße 14, 70469 Stuttgart, Germany 


\section{Introduction}

The new coronavirus (SARS-CoV-2) has become a global pandemic in the form of novel coronavirus pneumonia (COVID-19) in human, and influenced every aspect of human live. ${ }^{1}$ Statistical analysis showed that SARS-CoV-2 has infected subjects in 222 countries and districts, including China (a total of 117,501 confirmed cases and 5,343 deaths until June 18, 2021). ${ }^{2}$ The common symptoms of COVID-19 are fever, dry cough, and fatigue, and the main complications of this disease include acute respiratory distress syndrome, acute cardiac injury, and secondary infection. ${ }^{3}$ The number of cases has increased rapidly, however, with limited effective drugs targeting COVID-19 in the clinic. Thus, providing more clinical data and exploring treatment methods become urgently needed to block the severe epidemic situation of COVID-19.

Based on historical experience, research evidence, and current prevention programs, Traditional Chinese Medicine (TCM) is considered to be an effective and alternative means for COVID-19 prevention. ${ }^{4}$ Pudilanxiaoyan oral liquid (PDL), a well-known TCM preparation, is consisted of four herbs including Pu-gong-ying (Taraxacum mongolicum Hand.-Mazz. or Taraxacum borealisinense Kitam.), Huang-qin (Scutellaria baicalensis Georgi.), Ku-di-ding (Corydalis bungeana Turcz.), and Ban-lan-gen (Isatis indigotica Fort.). ${ }^{5}$ It has been commonly used for the treatment of viral infectious diseases including H1N1 and hand-foot-mouth disease. ${ }^{6,7}$ The effect of PDL on the treatment of viral infectious disease might be related to inhibition of viral replication and proliferation. An in vitro study revealed that the median effective concentrations $\left(\mathrm{EC}_{50}\right)$ of inhibiting respiratory syncytial virus (RSV) and adenoviruses serotype 3 strains were 28.08 and $28.10 \mathrm{mg} / \mathrm{L}$, respectively. ${ }^{8}$ Moreover, PDL has significant anti-inflammatory activity, which can relieve the overexpression of inflammatory factors caused by viral infection. ${ }^{9-11}$ PDL may also be one of the effective TCM drugs for COVID-19 treatment, which has been recommended by the Health Authority of Hainan Province in China. ${ }^{12} \mathrm{~A}$ recent research confirmed that PDL had a potent inhibitory effect against SARS-CoV-2, as well as relieved the pneumonia in SARS-CoV-2-infected hACE2 mice. ${ }^{13}$ So far, there is little research to elucidate the potential mechanism and active ingredients of PDL for COVID-19 treatment.

Network pharmacology is a new branch of bioinformatics that has emerged in recent years, ${ }^{14}$ which could be applied to rapidly discover the potential material basis and pharmacological mechanism of TCM based on the construction and analysis of the network of ingredient-target-pathway. ${ }^{15,16}$ In the present work, network pharmacology was applied to analyze the underlying material basis and mechanism of PDL for anti-COVID-19, with a view to provide more evidence choices for the clinical treatment of COVID-19. The flow chart to clarify the workflow is provided in - Fig. 1.

\section{Materials and Methods}

\section{Chemical and Biological Materials}

Compounds including curcumin, chrysin, cymaroside, luteolin, baicalin, baicalein, wogonin, apigenin, esculetin, oroxylin
A, and gallic acid were obtained from Chengdu Alfa Biotechnology Co., Ltd. (Chengdu, Sichuan, China). Lipopolysaccharide (LPS) and dimethyl sulfoxide were acquired from SigmaAldrich (Saint Louis, Missouri, United States). Fetal bovine serum (FBS) and Dulbecco's Modified Eagle Medium (DMEM) were purchased from Gibco (Auckland, New Zealand). AntiIKK $\alpha / \beta$ monoclonal antibodies (mAbs), anti-p-IKK $\alpha / \beta$ mAbs, anti-ERK1/2 mAbs, anti-p-ERK1/2 mAbs, anti-Akt mAbs, anti-p-Akt mAbs, anti-p-JNK mAbs, anti-JNK mAbs, and anti-GAPDH mAbs were obtained from Signalway Antibody (Baltimore, Maryland, United States).

\section{Collection of Ingredients in PDL and Target Prediction} At present, there are no reports on the separation and purification of chemical constituents from PDL, while only a UPLC-ESI-Orbitrap-MS/MS method was used to analyze and identify its chemical ingredients. ${ }^{17}$ Therefore, the ingredients involved in this work were derived from the analysis results of the article. The structures of these ingredients were drawn using ChemBioDraw Ultra 14.0 and saved as SMILES format for target prediction.

Two public databases, SwissTargetPrediction (http://www. swisstargetprediction.ch/) and STITCH (http://stitch.embl.de/ ), were used to predict related targets of ingredients from PDL. ${ }^{18-20}$ SwissTargetPrediction is an on-line tool for target prediction of small molecules and it has been widely used in 159 countries. In our present work, the value of probability, which is used to assess the accuracy of target predictions, was set to $\geq 0.5 .^{21}$ In STITCH database, confidence scores are assigned to interactions between compounds and proteins for reflecting their levels of significance and certainty. The compound-related targets were collected when their confidence scores are at least $0.7 .^{22}$

\section{Construction of PPI Network}

The compound-related targets were imported into the STRING database to obtain the PPI network. ${ }^{23}$ Parameters for STRING database were set as follows: the species is set to Homo sapiens, the minimum required interaction score is set to the highest confidence ( 0.9 ) and disconnected nodes in the network are hidden, and the remaining parameters are default. The analyzed result file in CSV format from the STRING database was downloaded and imported into Cytoscape 3.7.2 software. Then the key targets in the PPI network were identified and screened by NetworkAnalyzer in Cytoscape 3.7.2 software.

\section{Functional Analysis and Network Construction}

The key targets in the PPI network were imported into the Database for Annotation, Visualization, and Integrated Discovery (DAVID, https://david.ncifcrf.gov/summary.jsp) for the analysis of gene ontology (GO) function and Kyoto Encyclopedia of Genes and Genomes (KEGG) pathway enrichment. The KEGG pathways with $p<10^{-4}$ were regarded as the main pathways of PDL.

The network of potential active ingredient-target-pathway was established by Cytoscape 3.7.2 software. The nodes in the network represent the potential active ingredients, 


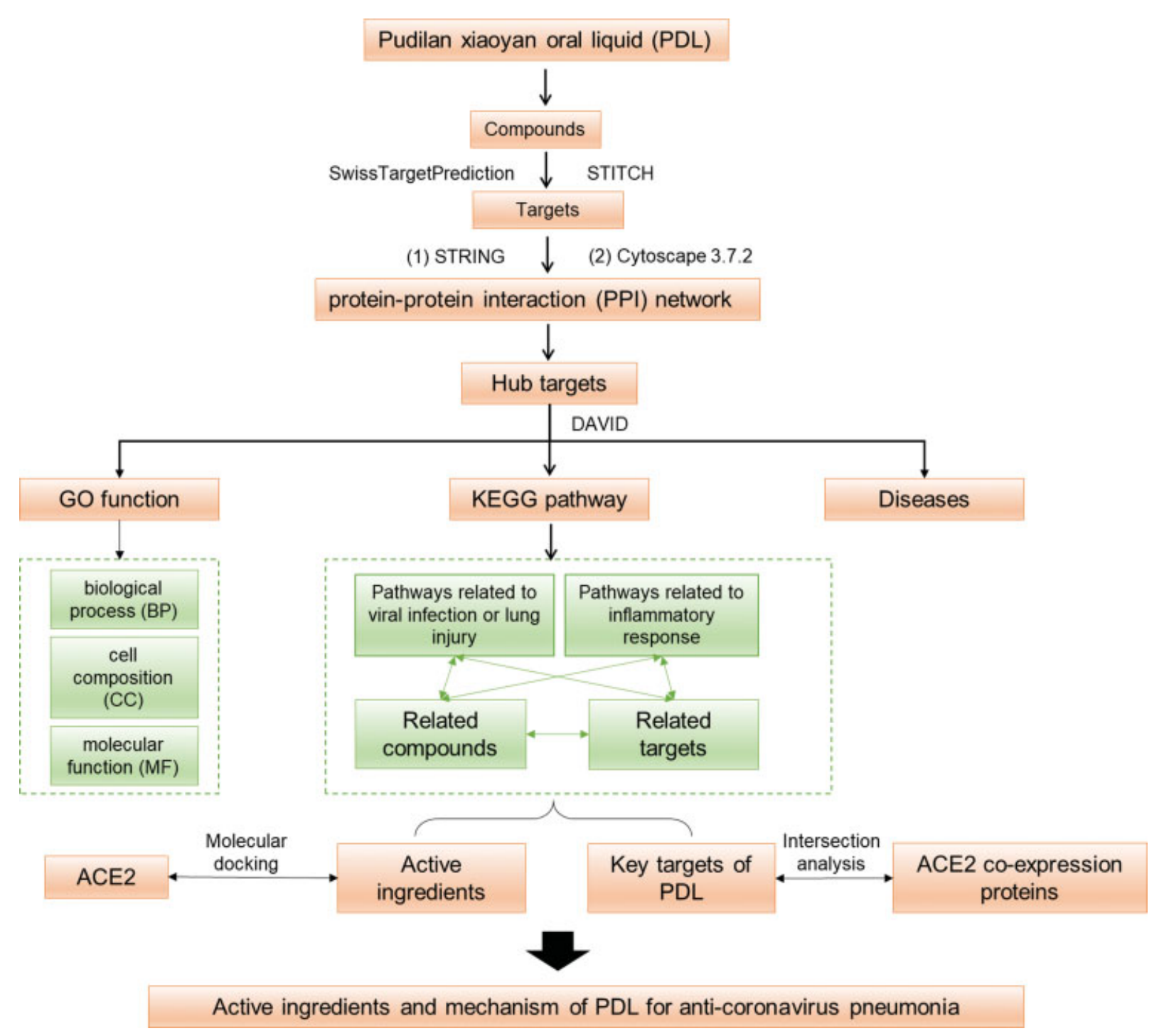

Fig.1 The workflow for investigating the active ingredients and mechanism of PDL for COVID-19 treatment. COVID-19, novel coronavirus pneumonia; PDL, pudilanxiaoyan oral liquid.

targets, or pathways, which would be connected by edges if they had interaction with others.

\section{Molecular Docking Study}

Recently, the structure of SARS-CoV-2 coronavirus 3CL hydrolase (Mpro) has been reported by Zihe Rao and Haitao Yang's research team and quickly became an important target for drug-screening researches. ${ }^{24}$ In addition, ACE2, a high-affinity-binding receptor of SARS-CoV-2 in the host cell, is also considered as an important target. ${ }^{25}$ Therefore, in the present study, the molecular docking study of the potential active ingredients from PDL was also performed on these two targets. First, based on the analysis results of the ingredienttarget-pathway network, the three-dimensional structures of the top 10 active ingredients would be downloaded from PubChem database (https://pubchem.ncbi.nlm.nih.gov/) in sdf format and then converted to pdb format by Open Babel (version 2.3.1). ${ }^{26}$ The solvent and ligands in the receptors of 1R4L and 6LU7 fetched from the Protein Data Bank (http:// www.rcsb.org/) would be removed by Pymol software. After adding the polar hydrogens and charges in both receptors and ingredients and saving in pdbqt formats, the docking processes were performed in AutoDock Vina. ${ }^{27}$ The visualization of docking results was also performed in Pymol.

\section{RAW264.7 Murine Macrophage Culture}

RAW264.7 murine macrophages were purchased from Shanghai Cell Bank, the Institute of Cell Biology, and Chinese Academy of Sciences (Shanghai, China) and were used for experiments not exceeding passages 10. RAW264.7 cells were maintained in DMEM containing $10 \% \mathrm{FBS}$ at $37^{\circ} \mathrm{C}$ in a moist atmosphere with $5 \% \mathrm{CO}_{2}$ and $95 \%$ air.

\section{NO Release Detection}

The effects of the tested compounds on LPS-induced nitric oxide (NO) production in RAW264.7 cells were determined using Griess reagent. RAW264.7 cells were seeded into 24well plates at $3 \times 10^{5}$ cells/well overnight. Cells were then pretreated with the tested compounds $(20 \mu \mathrm{mol} / \mathrm{L})$ or curcumin ( $5 \mu \mathrm{mol} / \mathrm{L}$, positive control) for 2 hours, followed by 
incubating with LPS $(1 \mu \mathrm{g} / \mathrm{mL})$ for an additional 24 hours. The supernatant of cell culture was then collected, and the NO Assay Kit (Beyotime, Shanghai, China) was used to detect NO production by RAW264.7 cells. The optical density of reaction solution was measured at $540 \mathrm{~nm}$ with a Multiskan FC Micropore reader (Thermo Fisher, United States).

\section{Western Blotting}

RAW264.7 cells were harvested and lysed with cold Radioimmunoprecipitation assay (RIPA) buffer containing $1 \%$ protease inhibitor and phosphatase inhibitor for 20 minutes to extract total protein on ice. After quantifying the concentration of the total protein, proteins with different molecular weights were separated by sodium dodecylsulphate polyacrylamide gel electrophoresis (SDS-PAGE) and transferred onto polyvinylidenefluoride (PVDF) membranes. After being blocked with $5 \%$ nonfat milk powder in Tris-buffered saline with Tween 20 (TBST), the PVDF membranes were blotted with specific primary antibodies overnight at $4^{\circ} \mathrm{C}$. The blotted PVDF membranes were then washed with TBST for three times and incubated with a horseradish peroxidaseconjugated secondary antibody at room temperature for 1 hour. Immunoblot signals were detected using chemiluminescence reagent (Beyotime, Shanghai).

\section{Results}

Ingredients of PDL and Its Corresponding Targets

In the present work, 59 compounds in PDL were collected and imported into the databases of SwissTargetPrediction and STITCH for target prediction. As a result, out of the 59 compounds, only 30 had targets that met the requirements in our work. The targets from the two databases were merged and a total of 190 targets were collected after eliminating duplicate targets. The ingredients and targets of PDL are shown in - Table S1 and - Table S2.

It was reported that ACE2, one of the major receptors that mediate the entry of SARS-CoV-2 into human cells, was coexpressed with 5,556 genes in the colon. The result of intersection analysis revealed that 68 of the 190 targets of PDL might be co-expressed with ACE2 (- Table S2).

\section{Construction and Analysis of PPI Network}

The ingredient-related targets of PDL were submitted to STRING for construction of PPI network and the result file was imported into Cytoscape 3.7.2 software for further analysis. In the PPI network, the node and edge represented target protein and interaction between protein-protein, respectively. In total, 146 nodes and 488 edges were included in the PPI network. The average node degree was 6.68 and the median was 5 . In the present work, the targets with a degree $\geq 7$ were determined as the key nodes of the PPI network and a total of 56 key targets were collected (- Table S2).

\section{GO Functional Annotation and KEGG Pathway Analysis of Target Proteins}

The GO terms, KEGG pathways' enrichment, and disease classes associated with the 56 key targets of the PPI network were determined in DAVID. As shown in - Fig. 2A, there were 388 GO entries $(p<0.05)$, including 300 biological process

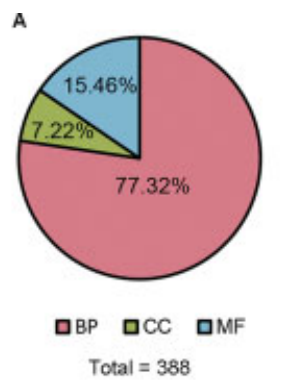

B

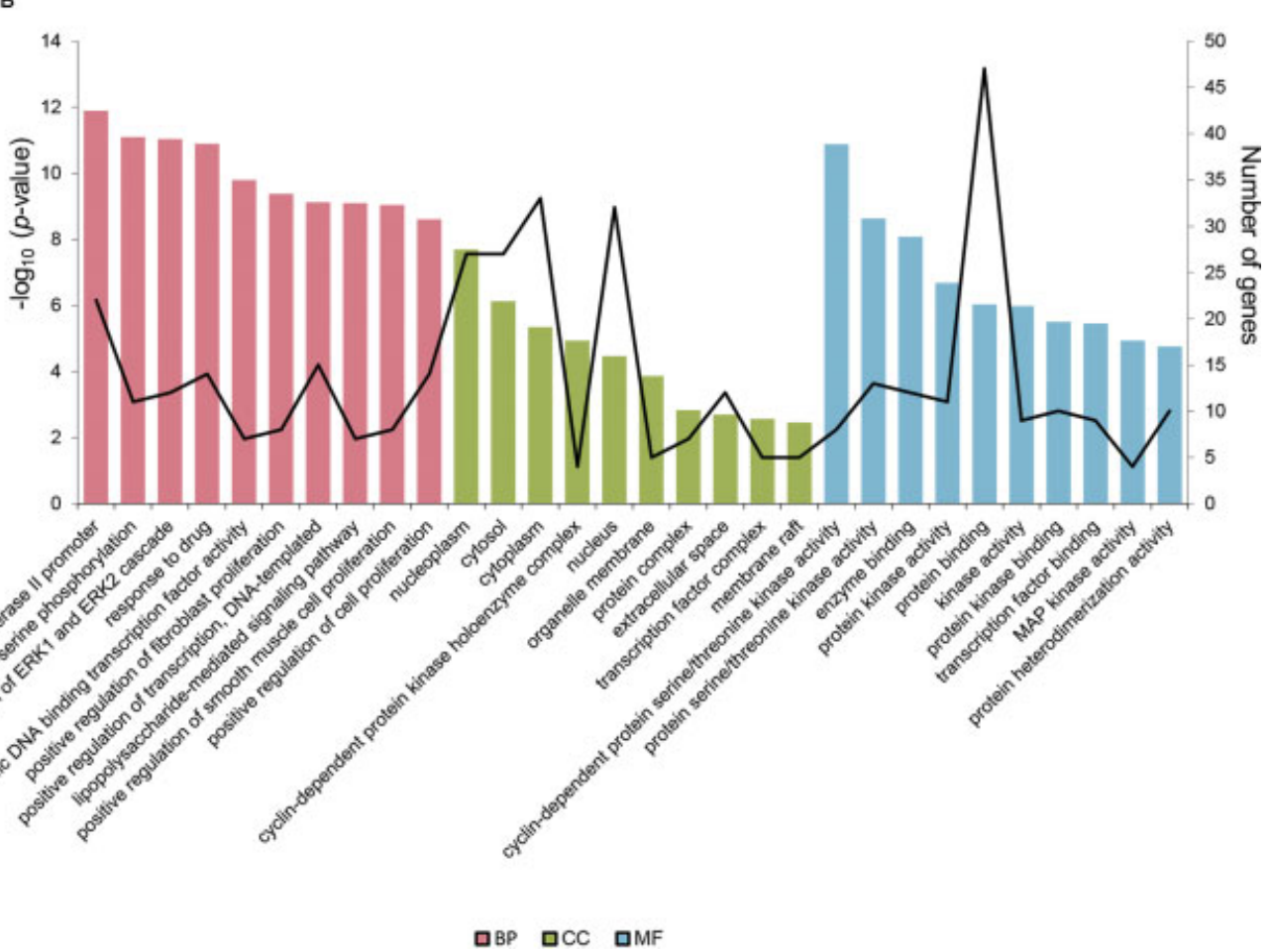

Fig. 2 GO enrichment analysis for 56 key targets of PDL. The order of importance was ranked from left to right by -log ${ }_{10}$ ( $p$-value) with bar chart. The number of targets sticks into each term with line chart. BP, biological process; CC, cell composition; GO, gene ontology; MF, molecular function; PDL, pudilanxiaoyan oral liquid. 
(BP) entries, 28 cell composition (CC) entries, and 60 molecular function (MF) entries, accounting for 77.3, 7.2, and $15.5 \%$, respectively. The top 10 entries of $\mathrm{BP}, \mathrm{CC}$, and MF are shown in -Fig. 2B. According to -Fig. 2B, the key targets of PDL mainly participated in positive regulation of transcription from RNA polymerase II promoter (GO: 0045944), peptidyl-serine phosphorylation (GO: 0018105), and positive regulation of ERK1 and ERK2 cascade (GO: 0070374). They were mainly located in nucleoplasm (GO: 0005654), cytosol (GO: 0005829), and cytoplasm (GO: 0005737). In addition, GO annotation demonstrated that the MFs of the key targets were mainly related to cyclin-dependent protein serine/threonine kinase activity (GO: 0004693), protein serine/threonine kinase activity (GO: 0004674), and enzyme binding (GO: 0019899).

In the results of KEGG pathway $\left(p<10^{-4}\right)$ was set as the cutoff criterion and 50 pathways were collected in which there are 24 pathways related to diseases and 12 are related to viral infection or lung injury, including legionellosis (hsa05134), tuberculosis (hsa05152), hepatitis C (hsa05160), hepatitis B (hsa05161), measles (hsa05162), influenza A (hsa05164), HTLV-I infection (hsa05166), herpes simplex infection (hsa05168), Epstein-Barr virus infection (hsa05169), viral carcinogenesis (hsa05203), small cell lung cancer (hsa05222), and nonsmall cell lung cancer (hsa05223). Of the 26 disease-free signal pathways, 14 are involved in regulating inflammatory responses, including signaling pathways of tumor necrosis factor (TNF; hsa04668), p53 (hsa04115), toll-like receptors (TLRs; hsa04620), PI3K-Akt (hsa04151), T cell receptor (hsa04660), NOD-like receptor (hsa04621), MAPK (hsa04010), FoxO (hsa04068), HIF-1 (hsa04066), B cell receptor (hsa04662), cAMP (hsa04024), neurotrophin (hsa04722), ErbB (hsa04012), and Fc epsilon RI (hsa04664). The above 26 pathways and involved genes are shown in -Fig. 3 and - Table 53. - Fig. 3B shows that PDL combated COVID-19 by regulating both TNF and PI3k/Akt signaling pathways. Among the targets of PDL, 14 and 18 targets are related to TNF and PI3k/Akt signaling pathways, respectively, and 5 of them are common targets of these two pathways. Both ACE2 and Mpro are considered as the potential targets of COVID-19 treatment, while Mpro is the key enzyme of coronaviruses, ${ }^{28}$ which is primed by TMPRSS2 and then uses ACE2 receptor to enter the host cell. ${ }^{29}$ Because both TMPRSS2 and ACE2 are the host targets in patients of COVID-19, they are selected for identification of the relationship with targets of PDL involved in pathways of TNF and PI3k/Akt signaling pathway. The above targets were imported into STRING database. The results were exported into Cytoscape software to map the network of their interactions. The interaction network of whole targets is shown in -Fig. 4A. Sub-network including anti-COVID-19 targets (ACE2 and TMPRSS2) and their directly connected nodes are displayed in - Fig. 4B. Our data suggest that the expression levels of AKT1, MAPK3, TP53, IL6, MYC, EGFR, and CDK4 might be influenced by ACE2 and TMPRSS2.

In addition, the clusters of diseases related to the key targets in PDL were also generated using functional annotation clustering in DAVID and the classification stringency was set as high. After clustering, 21 distinct clusters were obtained and are shown in -Table $\mathbf{5 4}$. The cluster with the highest enrichment level (the enrichment score: 7.58) was RSV bronchiolitis (false discovery rate $[\mathrm{FDR}]=1.77 \times 10^{-5}$ ). The 2nd cluster with the enrichment level with the enrichment score of 6.77 contains two diseases: inflammation/premature birth $\left(\mathrm{FDR}=9.31 \times 10^{-5}\right)$ and sarcoidosis $\left(\mathrm{FDR}=6.96 \times 10^{-4}\right)$. These diseases were related to viral infection or inflammation.

\section{Construction of Compound-Target Network}

According to KEGG pathway analysis by DAVID shown in - Table S3, a total of 30 targets were mapped into 12 pathways related to viral infection or lung injury and a total of 36 targets were involved into 14 pathways related to inflammation. There were 29 common targets in the two types of pathways. After merging these targets, 37 nonrepeating targets were collected for further analysis. First, 37 targets were imported into STRING to construct their PPI network
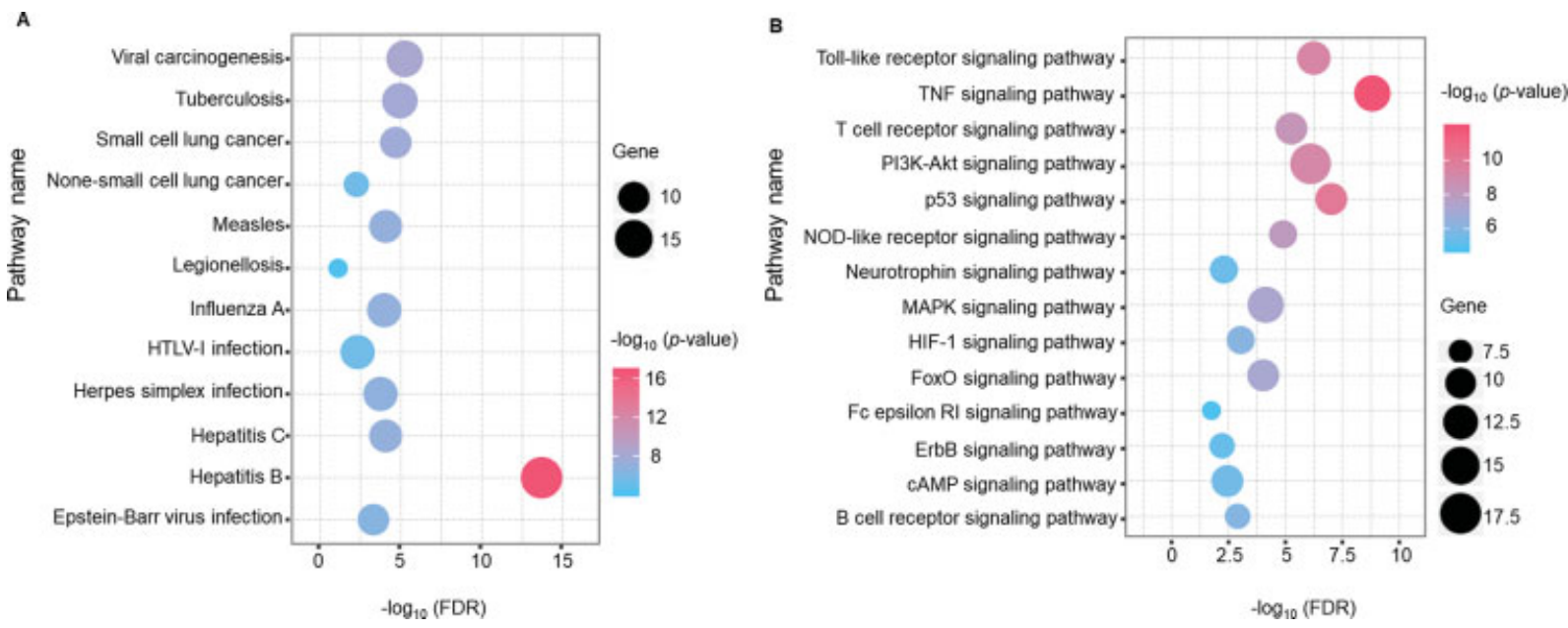

Fig. 3 KEGG pathway related with viral infection, lung injury, or inflammatory response of the key targets in PDL. The size of the bubbles in each bubble chart represents the gene counts in each pathway and the colors from red to blue represent the $p$-values from small to large. KEGG, Kyoto Encyclopedia of Genes and Genomes; PDL, pudilanxiaoyan oral liquid. 

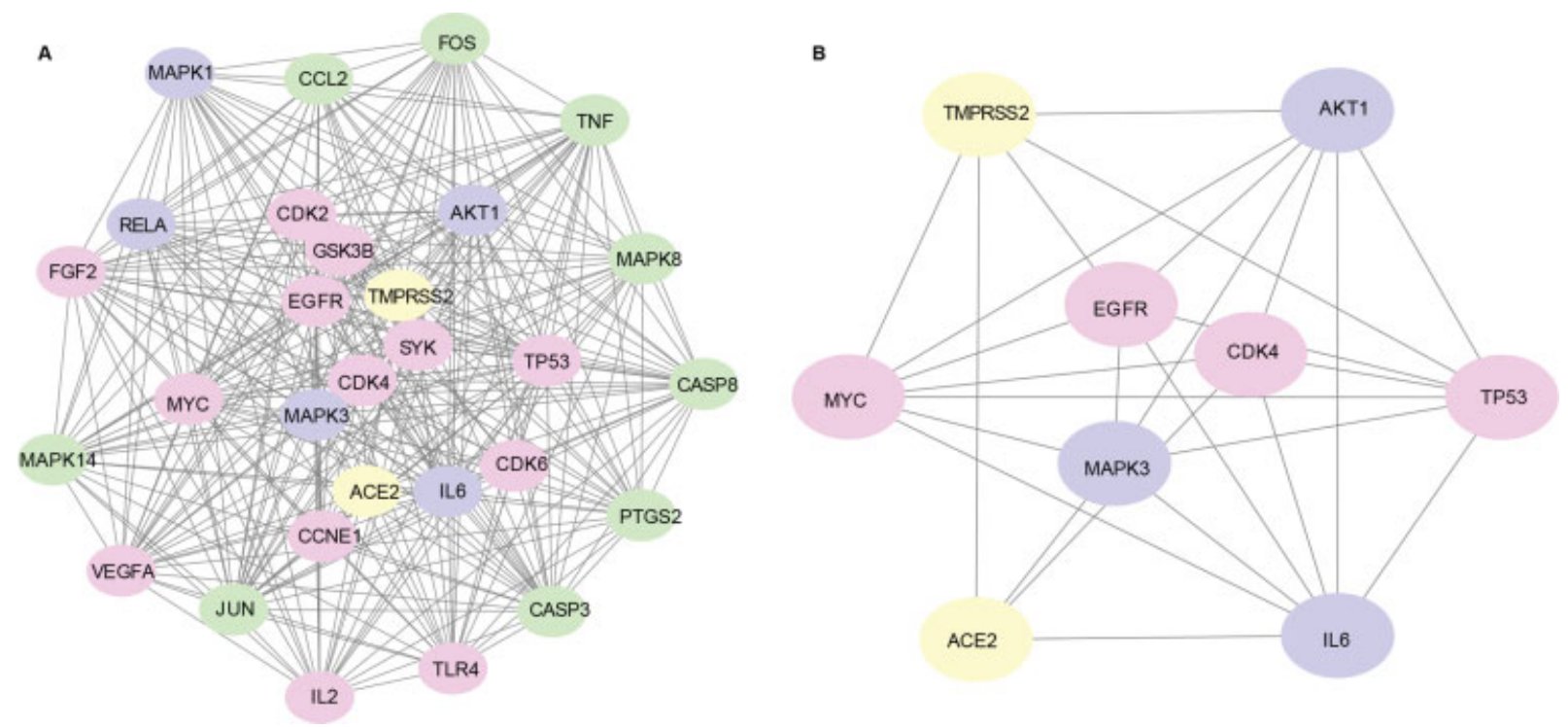

Fig. 4 The relationship of targets of PDL involved in the TNF and PI3k/Akt signaling pathway and anti-COVID-19 targets (ACE2 and TMPRSS2). Green nodes stand for genes involved in the TNF signaling pathway; red nodes stand for genes from PI3k/Akt signaling pathway; purple nodes stand for the common genes from TNF and PI3k/Akt signaling pathway; and yellow nodes stand for the anti-COVID-19 targets of ACE2 and TMPRSS2. PDL, pudilanxiaoyan oral liquid; TNF, tumor necrosis factor.

using the same parameters above and the result file was imported into Cytoscape 3.7.2 software. Then it was merged with the network constructed by nodes of compounds, targets, and signaling pathways, in which the nodes of compound-target and target-pathway were connected by edges (-Fig. 5).

In the above network, the impact of target on the entire network was evaluated by the degree obtained from the analysis result of the PPI network. The greater degree value of a node, the greater of its impact on the entire target network. To measure the regulation effect of a compound on the entire network, the regulation value of a compound was defined as the sum of the degree of all the targets related with the compound. For example, if a component was connected with two targets in the network and the degrees of these two targets in their PPI network are 10 and 5, respectively, the regulatory value of this component was 15 . The higher regulatory value of a compound, the more likely it is the more active substance in PDL. Based on the analysis results of the compound-target-pathway network, the top 10 targets and active ingredients that affect the entire network are shown in - Table 1 . Our data suggest that the top 10 active ingredients were mainly from Pu-gong-ying (apigenin, esculetin, oroxylin A, and gallic acid) and Huang-qin (luteolin, chrysin, baicalein, baicalin, wogonin, and cymaroside).

A protein-protein interaction network for the top 20 targets of PDL against COVID-19 was also constructed. The function modules of these 20 targets were then explored by MCODE in Cytoscape. - Fig. 6A shows two identified modules, which were marked in red and green, respectively. Based on GO enrichment analysis, BPs of module 1 (red) and module 2 (green) were mainly related to positive regulation of transcription from RNA polymerase II promoter (GO: 0045944) and peptidyl-serine phosphorylation (GO: 0018105), respectively, which were the top two BPs in the whole PPI network. KEGG pathway analysis $\left(p<10^{-4}\right)$ showed 16 and 26 pathways identified for module 1 and module 2, respectively. There were eight common pathways, which were hepatitis B (hsa05161), T cell receptor (hsa04660), Chagas disease (American trypanosomiasis) (hsa05142), pathways in cancer (hsa05200), pertussis (hsa05133), TLR (hsa04620), TNF (hsa04668), and PI3K-Akt (hsa04151), which were related to viral infection or inflammatory response (-Fig. 6B).

\section{Effects of Top 10 Active Ingredients on NO Release and Their Possible Anti-inflammatory Mechanism}

Network pharmacology analysis showed that anti-inflammatory activity might be one of the most important mechanisms, whereby PDL exerted its anti-COVID-19 effect; therefore, the anti-inflammatory activities and possible mechanisms of top 10 active ingredients from PDL were investigated. NO has been shown to be a valuable biomarker for determining the extent of inflammation. To initially explore the anti-inflammatory activity of 10 active ingredients, RAW264.7 cells were treated with the test compounds $(20 \mu \mathrm{mol} / \mathrm{L})$ or curcumin $(5 \mu \mathrm{mol} / \mathrm{L}$, positive control) for 2 hours, then incubated with LPS $(1 \mu \mathrm{g} / \mathrm{mL})$ for an additional 24 hours. The level of NO was measured using Griess reagent. As shown in - Fig. 7A, compared with the blank control group, LPS treatment led to considerable increase of NO release in RAW264.7 cells, and the increase in LPS-induced NO release was significantly reversed by chrysin, cymaroside, luteolin, baicalin, baicalein, wogonin, apigenin, esculetin, and oroxylin $\mathrm{A}(\boldsymbol{p}<0.0001$ or $p<0.01)$.

To investigate the possible anti-inflammatory mechanisms, Western blotting was conducted to assess the effects of luteolin and baicalein on the activation of IKK $\alpha / \beta$, ERK $1 / 2$, Akt, and JNK. - Fig. 7C shows that both luteolin and baicalein significantly decreased the phosphorylation of IKK- $\alpha / \beta$ 


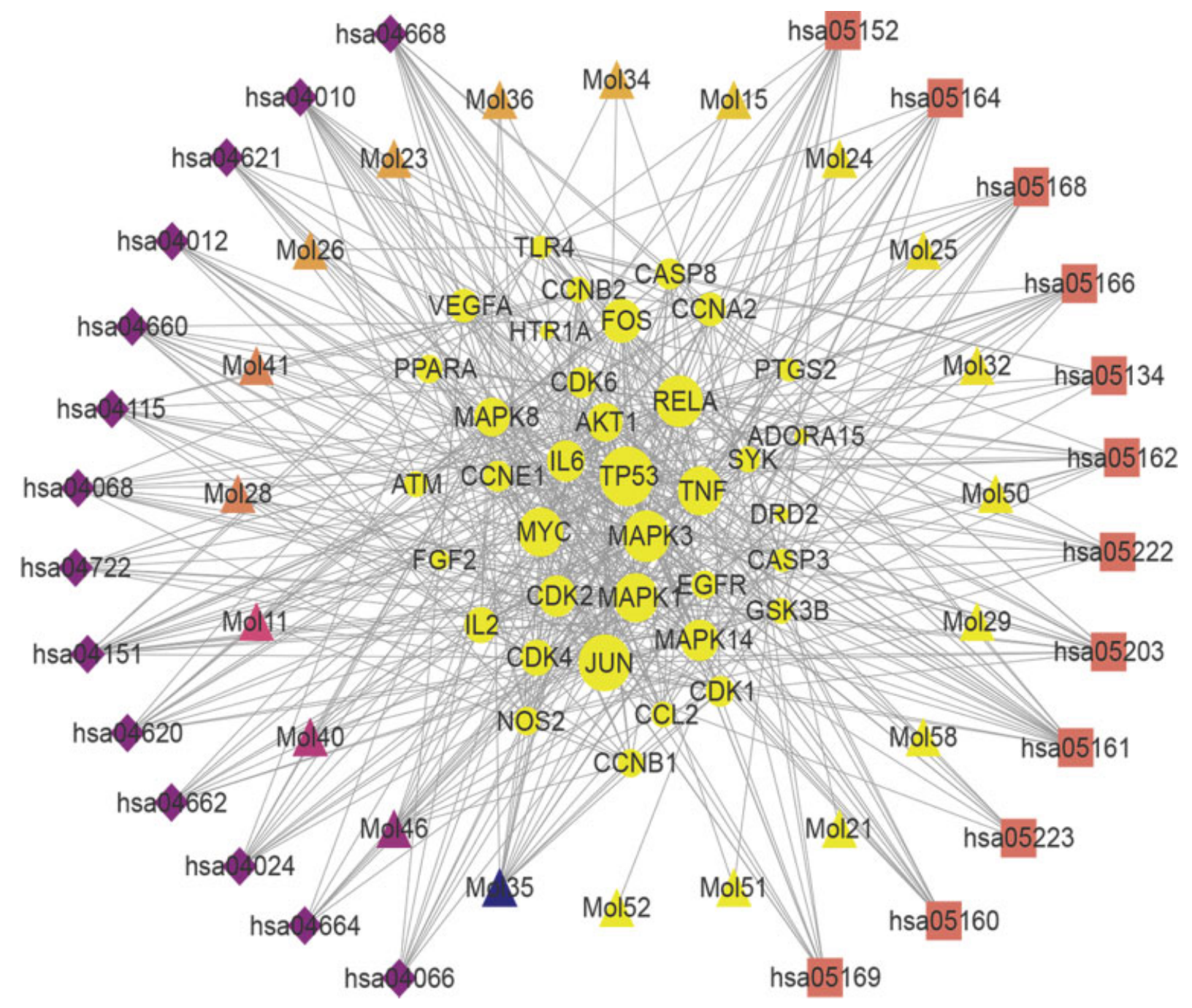

Fig. 5 The compound-target-pathway network of PDL. Pathways with purple diamond belong to pathway 1, which represents the pathways related with viral infection or lung injury, while pathways with brown square belong to pathway 2 , which represents the pathways involved in regulating the inflammatory response. PDL, pudilanxiaoyan oral liquid.

Table 1 The top 10 targets and active ingredients that affect the entire network

\begin{tabular}{|l|l|l|l|}
\hline Targets & Degree & Ingredients & $\begin{array}{l}\text { Regulation } \\
\text { value }\end{array}$ \\
\hline TP53 & 19 & Luteolin (Mol 35) & 129 \\
\hline JUN & 18 & Apigenin (Mol 46) & 83 \\
\hline MAPK3 & 16 & Esculetin (Mol 40) & 74 \\
\hline RELA & 16 & Chrysin (Mol 11) & 64 \\
\hline MAPK1 & 15 & Baicalein (Mol 28) & 44 \\
\hline MYC & 15 & Oroxylin A (Mol 41) & 43 \\
\hline TNF & 15 & Baicalin (Mol 26) & 34 \\
\hline FOS & 13 & Wogonin (Mol 23) & 33 \\
\hline CDK2 & 12 & Cymaroside (Mol 36) & 32 \\
\hline MAPK14 & 12 & Gallic acid (Mol 34) & 29 \\
\hline IL6 & 12 & & \\
\hline
\end{tabular}

induced by LPS, while they had no effect on the phosphorylation of other target proteins.

\section{Molecular Docking Result}

In the present work, the molecular docking studies of the following compounds were performed, including the top 10 active ingredients from PDL and drug candidates for COVID19 (remdesivir, ${ }^{30}$ ribavirin, ${ }^{31}$ nitazoxanide, ${ }^{32}$ favipiravir, ${ }^{32}$ and chloroquine ${ }^{30}$ ). - Table 2 shows that among the drug candidates, remdesivir had the highest affinities to Mpro (6LU7) and ACE2 receptor (1R4L), and its total docking score was $-14.6 \mathrm{kcal} / \mathrm{mol}$. Among the top 10 active ingredients from PDL, all of them had high affinities to Mpro and ACE2 receptor (docking score $<-5 \mathrm{kcal} / \mathrm{mol}$ ) and some of the active ingredients had higher docking score than remdesivir, including luteolin, baicalein, baicalin, and cymaroside. Baicalin, the total docking score could reach $-17.1 \mathrm{kcal} / \mathrm{mol}$, could be considered as the most active compound in PDL. This result partly provides the rationale for using baicalin as the quality marker of PDL in the Chinese Pharmacopoeia (2020 edition). In the Chinese Pharmacopoeia, the content of 

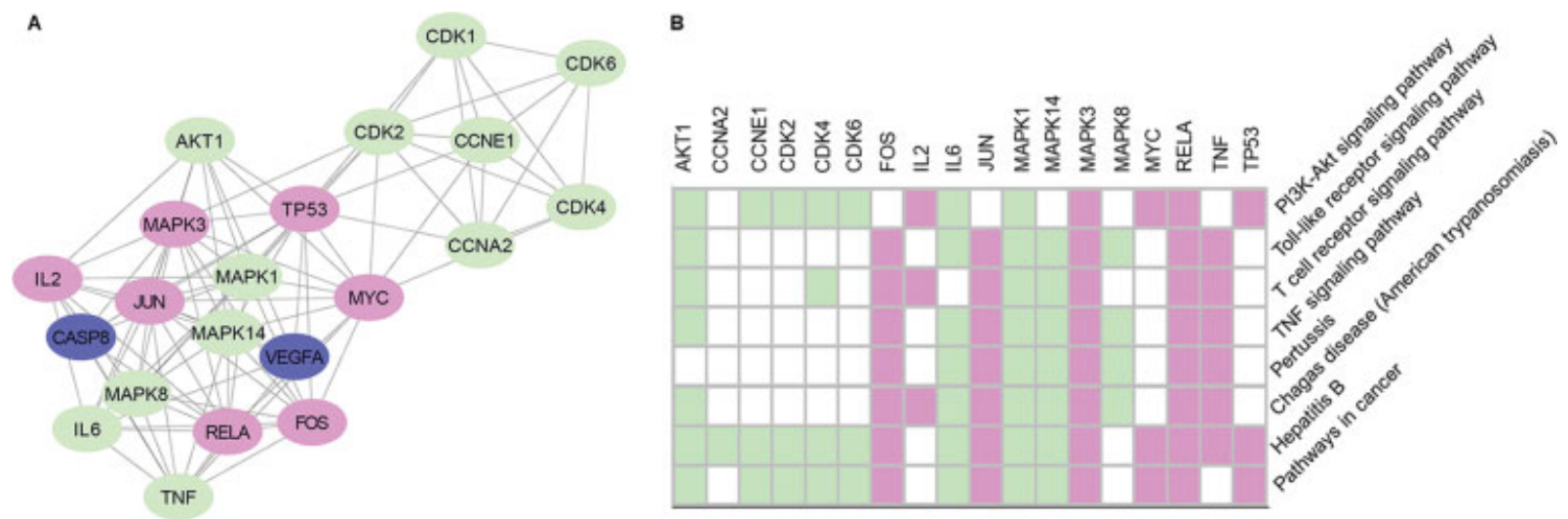

Fig. 6 The protein-protein interaction network and pathways of top targets of PDL against COVID-19. PDL, pudilanxiaoyan oral liquid.

A

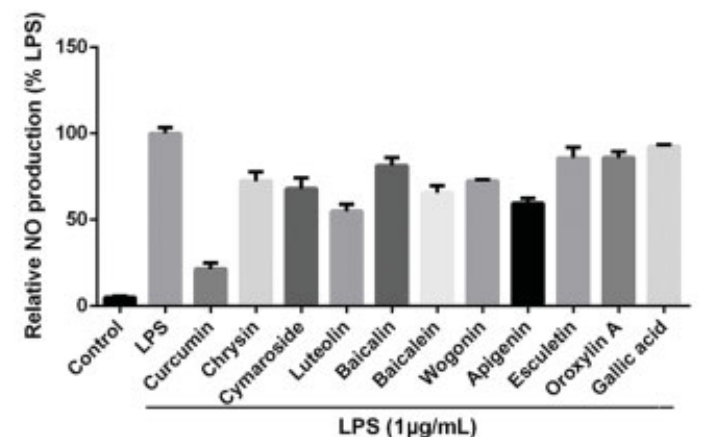

B

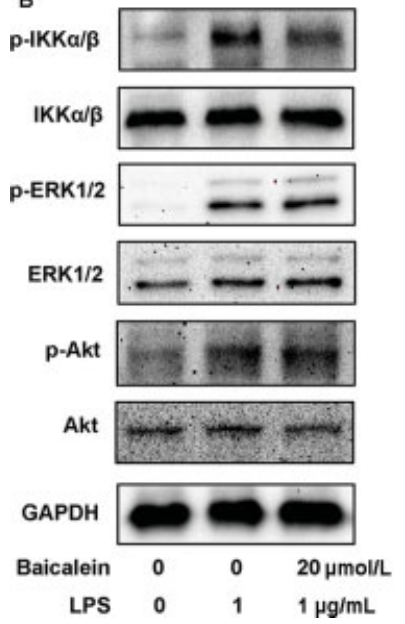

Fig. 7 The anti-inflammatory effects of compounds on LPS-activated RAW264.7 macrophages. (A) The effects of compounds on LPSinduced NO production in RAW264.7 cells. Data are presented as mean \pm standard deviation (SD), $n=3 .{ }^{*} p<0.05,{ }^{* *} p<0.01,{ }^{* * *} p$ $<0.001,{ }^{* * * *} p<0.0001$ versus LPS. The effects of baicalein (B) and luteolin (C) on LPS-induced activation of inflammatory signaling pathways in RAW264.7 cells. LPS, lipopolysaccharide.

baicalin in PDL was required to be higher than $6.0 \mathrm{mg} / \mathrm{mL}$. As reported by Ruan et al, the content of baicalin in PDL can reach $\sim 6.3 \mathrm{mg} / \mathrm{mL}$, which also might be the ingredient with the highest content in PDL. ${ }^{33}$ As shown in - Fig. 8, the key residues in 1R4L for baicalin binding were ASP-383 and SER47, while ARG-131, GLU-290, GLU-288, LYS-137, and LYS-5 were the key residues in 6LU7.
Table 2 The molecular docking results

\begin{tabular}{|l|l|l|l|}
\hline \multirow{2}{*}{ Ligand } & \multicolumn{3}{l|}{ Docking score (kcal/mol) } \\
\cline { 2 - 4 } & 6 LU7 & 1 R4L & SUM \\
\hline Luteolin & -6.9 & -8 & -14.9 \\
\hline Apigenin & -6.7 & -7.6 & -14.3 \\
\hline Esculetin & -5.5 & -6.7 & -12.2 \\
\hline Chrysin & -6.7 & -7.6 & -14.3 \\
\hline Baicalein & -6.7 & -8.1 & -14.8 \\
\hline Oroxylin A & -6.6 & -7.7 & -14.3 \\
\hline Baicalin & -7.5 & -9.6 & -17.1 \\
\hline Wogonin & -6.5 & -7.8 & -14.3 \\
\hline Cymaroside & -7.8 & -9.1 & -16.9 \\
\hline Gallic acid & -5.2 & -6.1 & -11.3 \\
\hline Remdesivir & -6.4 & -8.2 & -14.6 \\
\hline Ribavirin & -6.3 & -6.9 & -13.2 \\
\hline Nitazoxanide & -5.8 & -7 & -12.8 \\
\hline Favipiravir & -5.2 & -5.6 & -10.8 \\
\hline Chloroquine & -5.5 & -6.5 & -12 \\
\hline
\end{tabular}

\section{Discussion}

PDL is a common TCM prescription composed of four herbs including Pu-gong-ying, Huang-qin, Ku-di-ding, and Banlan-gen. Huang-qin is the fourth frequently used herb in TCM for prevention and treatment of COVID-19. Interestingly, Pu-gong-ying is also an important herb in Reyanning mixture, another drug for COVID-19 treatment. ${ }^{34-36}$ The protein expression of iNOS, TNF- $\alpha$, interleukin-6 (IL-6), and IL-1 $\beta$ could been suppressed by Ku-di-ding, ${ }^{37}$ and the 3CLpro could also been inhibited by Ban-lan-gen. ${ }^{38}$ In this work, network pharmacology tools were mainly applied to explore the potential active constituents and pharmacological mechanism of PDL for anti-COVID-19.

Our results first suggest that the main targets of PDL were TP53, JUN, MAPK3, RELA, MAPK1, MYC, TNF, FOS, CDK2, MAPK14, and IL-6 (-Table 1). Evidence suggested that the 

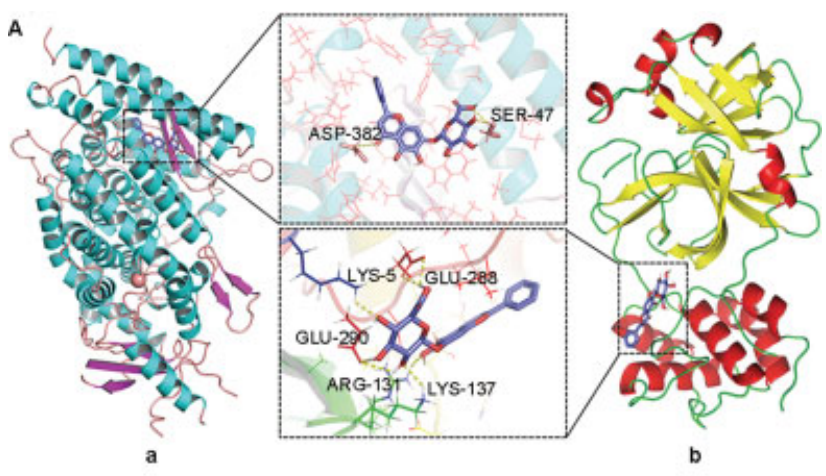

B

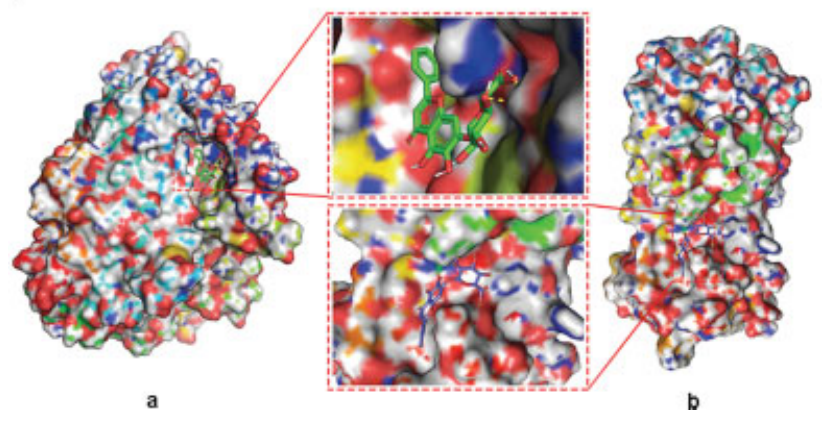

Fig. 8 The three-dimensional cartoon display of interactions between baicalin and receptors. (A) 1R4L, ACE2 receptor. (B) 6LU7, SARS-CoV-2 main protease.

activation of JUN plays a key role in the development of coronavirus infection, ${ }^{39}$ which further confirmed the antiCOVID-19 effect of PDL at a molecular level. In the top 10 targets, only MAPK3 was co-expressed with ACE2. MAPK3, also known as ERK1, is an essential node in the MEK/ERK signaling pathway. An overwhelming amount of research studies have demonstrated that the MEK/ERK pathway could be activated by RNA viruses, including coronaviruses, resulting in virus replication and expression of proinflammatory cytokine. ${ }^{40-42}$ Patients with severe COVID-19 are commonly companied with enhancement in the release of proinflammatory cytokines and chemokines characterized as cytokine storm, which might be associated with shock, widespread tissue damage, and progressive multiple organ failure. ${ }^{3,43-46}$ Inspired by this, we inferred that the activation of ERK may be one of the important factors leading to cytokine storm in the patients of COVID-19. ACE2 is an import cellular receptor of SARS-CoV-2 and the high expression of ACE2 are associated with increased severity of COVID-19 illness, ${ }^{47}$ but after SARS-CoV-2 infection, the level of ACE2 protein is greatly reduced, resulting in angiotensin II accumulation followed by the activation of AT1R and subsequent ERK phosphorylation, ${ }^{48,49}$ leading to the release of proinflammatory cytokines, as mentioned above. Hence, the co-expression between ACE2 and MAPK3 may be related with the abundance of proinflammatory cytokines.

Targets of PDL could be mapped into 12 pathways related with viral infection or lung injury, indicating that PDL might have anti-COVID-19 activity by directly acting on key host targets or targets involved the crucial pathways of coronavirus infection process. Interestingly, 29 out of 30 targets in the pathways associated with viral infection or lung injury were included in the pathways associated with inflammatory reaction. On the one hand, it may be explained that the activation of signaling pathways in coronavirus infection could also induce activation of inflammatory responses, and both contribute to the pathogenesis of COVID- $19 .{ }^{50}$ On the other hand, it also reflects that anti-COVID-19 of PDL may partly be attributed to its anti-inflammatory activity. ${ }^{10}$ Our data revealed the top targets of PDL against COVID-19, including AKT1, FOS, JUN, CDK2, CDK4, CDK6, CCNE1, IL2, IL6, MAPK3, MAPK1, MAPK8, MYC, RELA, MAPK14, P53, TNF, CASP8, CDK1, CCNA2, and VEGFA, were mainly involved in signaling pathways of PI3K-Akt (hsa04151), TNF (hsa04668), TLR (hsa04620), etc. (- Fig. 6). Furthermore, - Fig. 9 shows a diagram of proposed mechanisms with key targets of PDL for its anti-COVID-19 activity. As is well known, SARS-CoV-2, primed by TMPRSS2, uses ACE2 for entry. After replication in host cells, new SARS-CoV-2 is produced, then ACE2 levels are decreased in SARS-CoV-2-infected cells leading to increase in AT-II, resulting the activation of AT1R. ${ }^{29}$ Activated AT1R can lead to massive expression of proinflammatory cytokines through the ERK and p38 signaling pathway. ${ }^{51}$ On the other hand, SARS-CoV-2 can also activate the immune inflammatory response through the TLR pathway. Our data suggested the anti-COVID-19 mechanisms of PDL, shown in red in - Fig. 9.

We also investigated the main active ingredient of PDL in COVID-19 therapy, and first suggested top 10 active ingredients with possible anti-inflammatory mechanisms. As we all know, baicalin is a main active ingredient in Huang-qin ( $S$. baicalensis Georgi.), which is the fourth frequently used herb in TCM for COVID-19 prevention and treatment. ${ }^{34}$ Our result is consistent with the previous reports that baicalin was an important ingredient for COVID-19 treatment, identified by docking screening $24,52,53$ or an in vitro study for anti-SARSCoV-2 assay. ${ }^{54}$ However, comparing the two results from - Tables $\mathbf{1}$ and 2, it can be found that baicalin might not be the most critical component of PDL against COVID-19. The regulation value of baicalin is only 34 , which is ranked seventh among all compounds in PDL. The possible explanations for this difference might be as follow: the results of $\boldsymbol{- T a b l e s} \mathbf{1}$ and $\mathbf{2}$ illustrate different perspectives on the activity of PDL against COVID-19. In - Table 2, only the influence of a single component on a single key target is considered; however, in - Table 1, the influences of single component to multitargets or multicomponents to single targets are all included. In 2020, Zhou et al reported that there are at least 119 host proteins associated with COVID$19^{55}$; however, by considering the synergistic effects of compounds on multiple targets (a pervasive mechanism of TCM in disease treatment), our result may be more accurate. ${ }^{56-59}$

In the present work, although the potential active constituents and pharmacological mechanisms of PDL for antiCOVID-19 have been demonstrated by network pharmacology, it also has certain limitations. At present, in the network pharmacology research studies of many TCM preparations, ${ }^{60-62}$ the ingredients of a preparation are often equivalent to the sum of ingredients from each herb contained in 


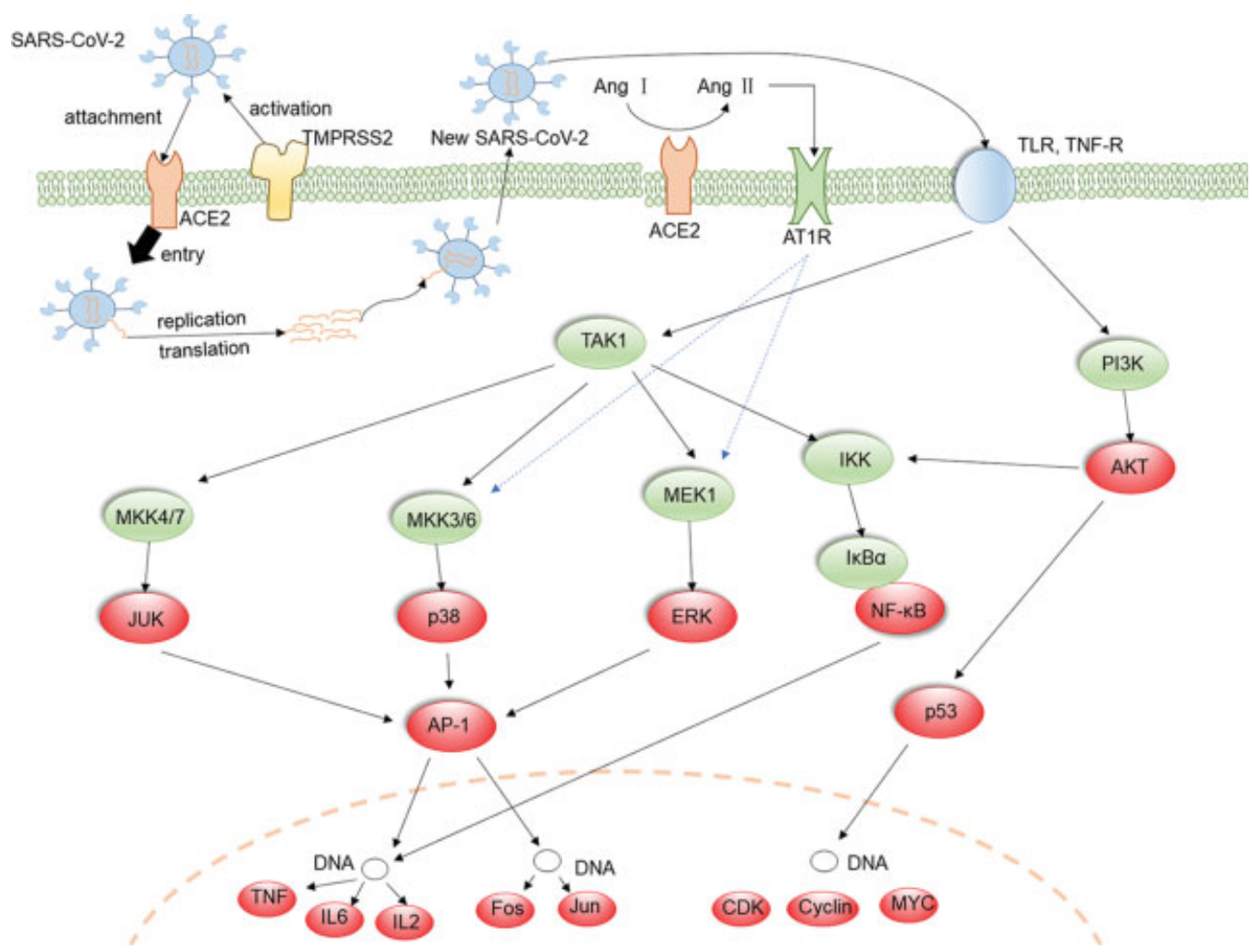

Fig. 9 Proposed mechanisms with key targets underlying the suppressive effects of PDL on COVID-19. PDL, pudilanxiaoyan oral liquid.

this preparation. It is not an accurate way because the ingredient losses from herbs during extraction process are not taken into account. To overcome this deficiency, the ingredients used in this work were directly collected from an UPLC-ESI-Orbitrap-MS/MS analysis report of PDL. But this method still has a problem because a large percentage of MS signals could not be identified due to the shortcomings of UPLC-MS/MS analysis technology. ${ }^{63}$ Therefore, further studies are required for structure identification by different methods. Second, the anti-inflammatory effects of top 10 ingredients were investigated on LPS-activated RAW264.7 macrophages in this work. - Fig. 7 demonstrates that the top 10 ingredients except for gallic acid could reduce the release of NO, further confirming that PDL might be a potential drug for COVID-19 treatment by the inhibition of the cytokine storm. However, the ingredients of baicalein and luteolin, which had higher anti-inflammatory activity than others from PDL, could significantly increase the IKK $\alpha / \beta$ expression. Up to now, plenty of studies have suggested that the increase of $I K K \alpha / \beta$ expression could reduce the release of NF-KB, which can induce the transcription of different cytokines in the nucleus. ${ }^{64}$ Previous experiments have also confirmed that the activation of the NF- $\mathrm{KB}$ signaling pathway is one of the important factors in the SARS-CoV-2-induced inflammatory storm ${ }^{65}$; therefore, we inferred that inhibiting the activity of the NF-KB signaling pathway is a mechanism of PDL for COVID-19 treatment. But in this work, we also found that the top active ingredients of PDL had no significant effects on the expression of ERK1/2, Akt, and JNK, which were the top targets of PDL according to the results of network pharmacology analysis. The possible explanation for this might be attributed to differences in evaluation criteria. In cell experiments, the activity of a compound was determined by its affinity with certain targets, while in the network pharmacology analysis, the top 10 targets were screened by the parameter of "degree"; the higher value of top targets suggested that the targets could interact with more ingredients, indicating strong synergy. But it does not imply that the target had higher affinity with each compound. More experimental investigations in animals or in the clinic are needed to confirm the effects of PDL for COVID-19 treatment in future.

\section{Conclusions}

In the present study, the potentially active constituents and molecular mechanisms of PDL against COVID-19 were examined with the combination of network pharmacology and experimental verification. The results from network pharmacology revealed that the mechanisms of PDL against COVID-19 were mainly attributable to pathways related to viral infection, lung injury, or inflammatory responses. Moreover, the key active ingredients in PDL were luteolin, apigenin, esculetin, chrysin, baicalein, oroxylin A, baicalin, wogonin, cymaroside, and gallic acid. Further experiments in vitro confirmed the anti-inflammatory effect of these active ingredients, which may relate to their inhibiting effect on the NF-KB signaling pathway. As a result, our findings provide preliminary evidence for PDL in the treatment of COVID-19. 


\section{Abbreviations for the Targets}

$\begin{array}{ll}\text { ACE2 } & \text { Angiotensin-converting enzyme } 2 \\ \text { AKT1 } & \text { RAC- } \alpha \text { serine/threonine-protein kinase } \\ \text { AT1R } & \text { Angiotensin II receptor type 1 } \\ \text { CASP8 } & \text { Caspase } 8 \\ \text { CCNA2 } & \text { Cyclin A2 } \\ \text { CCNE1 } & \text { Cyclin E1 } \\ \text { CDK1 } & \text { Cyclin-dependent-like kinase 1 } \\ \text { CDK2 } & \text { Cyclin-dependent-like kinase } 2 \\ \text { CDK4 } & \text { Cyclin-dependent-like kinase } 4 \\ \text { CDK6 } & \text { Cyclin-dependent-like kinase 6 } \\ \text { EGFR } & \text { Epidermal growth factor receptor } \\ \text { ERK1/2 } & \text { Extracellular signal-regulated protein kinase } \\ \text { FOS } & \text { Proto-oncogene c-Fos } \\ \text { GSCF } & \text { Granulocyte colony-stimulating factor } \\ \text { IL2 } & \text { Interleukin 2 } \\ \text { IL6 } & \text { Interleukin 6 } \\ \text { JNK } & \text { c-Jun N-terminal kinase } \\ \text { JUN } & \text { Transcription factor AP-1 } \\ \text { MAPK1 } & \text { Mitogen-activated protein kinase 1 } \\ \text { MAPK14 } & \text { Mitogen-activated protein kinase 14 } \\ \text { MAPK3 } & \text { Mitogen-activated protein kinase 3 } \\ \text { MAPK8 } & \text { Mitogen-activated protein kinase } 8 \\ \text { MYC } & \text { Myc proto-oncogene protein } \\ \text { RELA } & \text { Transcription factor p65 } \\ \text { TMPRSS2 } & \text { Transmembrane serine protease 2 } \\ \text { TNF } & \text { Tumor necrosis factor } \\ \text { TP53 } & \text { Cellular tumor antigen p53 } \\ \text { VEGFA } & \text { Vascular endothelial growth factor A } \\ & \end{array}$

\section{Funding}

This research was funded by the Development Project of Shanghai Peak Disciplines-Integrative Medicine (Grant No. 20180101), the Special Project of International Technology Cooperation of One Belt and One Road (Grant No. 2017C04009), and the National Key Research and Development Program of China (Grant No. 2017YFC1703903).

\section{Conflict of Interest}

The authors declare that they have no competing interests.

\section{References}

1 Lazarus JV, Ratzan SC, Palayew A, et al. A global survey of potential acceptance of a COVID-19 vaccine. Nat Med 2021;27(02): $225-228$

2 Coronavirus WHO. (COVID-19) Dashboard. China. Accessed July, 2021 at: https://covid19.who.int/region/wpro/country/cn

3 Wang D, Hu B, Hu C, et al. Clinical characteristics of 138 hospitalized patients with 2019 novel coronavirus-infected pneumonia in Wuhan, China. JAMA 2020;323(11):1061-1069

4 Luo H, Tang QL, Shang YX, et al. Can Chinese medicine be used for prevention of corona virus disease 2019 (COVID-19)? A review of historical classics, research evidence and current prevention programs. Chin J Integr Med 2020;26(04):243-250

5 Committee NP. Chinese Pharmacopoeia. Beijing: Chemical Industry Press; 2015
6 Yi Y. The effect of Pudilan Xiaoyan oral liquid (PDL) on the treatment of herpes simplex ulcerative esophagitis influenza $A$ (H1N1). Mod J Integr Tradit Chin West Med 2010;19:2925-2926

7 Wang LX, Miao Q Xie YM, et al. Expert consensus statement on Pudilan Xiaoyan Oral Liquid in clinical practice [in Chinese]. Zhongguo Zhongyao Zazhi 2019;44(24):5277-5281

8 Xuan W, Li Y, Tao H, et al. Antiviral effects of pudilan xiaoyan oral liquid on respiratory syncytial virus and adenovirus in vitro. J Pract Med 2015;31:1838-1840

9 Feng L, Yang N, Li C, et al. Pudilan xiaoyan oral liquid alleviates LPS-induced respiratory injury through decreasing nitroxidative stress and blocking TLR4 activation along with NF-KB phosphorylation in mice. J Ethnopharmacol 2018;214:292-300

10 Tian G, Li C, Zhai Y, et al. GC-MS based metabolomic profiling of lung tissue couple with network pharmacology revealed the possible protection mechanism of Pudilan Xiaoyan oral liquid in LPS-induced lung injury of mice. Biomed Pharmacother 2020;124:109833

11 Jin Y, Lin X, Song L, et al. The effect of pudilan anti-inflammatory oral liquid on the treatment of mild recurrent aphthous ulcers. Evid Based Complement Alternat Med 2017;2017:6250892

12 Rujun S, Yuyu H, Xikun S, et al. Discussion on rational use of chinese patent medicine recommended from diagnosis and treatment protocols for COVID-19. Chin J Mod Appl Pharm 2020; 37:782-787

13 Deng W, Xu Y, Kong Q et al. Therapeutic efficacy of Pudilan Xiaoyan Oral Liquid (PDL) for COVID-19 in vitro and in vivo. Signal Transduct Target Ther 2020;5(01):66

14 Hopkins AL. Network pharmacology: the next paradigm in drug discovery. Nat Chem Biol 2008;4(11):682-690

15 Cui S, Chen S, Wu Q Chen T, Li S. A network pharmacology approach to investigate the anti-inflammatory mechanism of effective ingredients from Salvia miltiorrhiza. Int Immunopharmacol 2020;81:106040

16 Zhang J, Liang R, Wang L, Yang B. Effects and mechanisms of Danshen-Shanzha herb-pair for atherosclerosis treatment using network pharmacology and experimental pharmacology. J Ethnopharmacol 2019;229:104-114

17 Zhao LJ, Gao WY, Gu XR, Wang HJ, Zhao HY, Bian BL. Identification and attribution of chemical compounds of Pudilan Antiphlogistic Oral Liquid [in Chinese]. Zhongguo Zhongyao Zazhi 2019;44(08):1573-1587

18 Daina A, Michielin O, Zoete V. SwissTargetPrediction: updated data and new features for efficient prediction of protein targets of small molecules. Nucleic Acids Res 2019;47(W1):W357-W364

19 Gfeller D, Grosdidier A, Wirth M, Daina A, Michielin O, Zoete V. SwissTargetPrediction: a web server for target prediction of bioactive small molecules. Nucleic Acids Res 2014;42(Web Server issue):W32-W38

20 Kuhn M, von Mering C, Campillos M, Jensen LJ, Bork P. STITCH: interaction networks of chemicals and proteins. Nucleic Acids Res 2008;36(Database issue):D684-D688

21 Zeng Q, Li L, Siu W, et al. A combined molecular biology and network pharmacology approach to investigate the multi-target mechanisms of Chaihu Shugan San on Alzheimer's disease. Biomed Pharmacother 2019;120:109370

22 Kuhn M, Szklarczyk D, Franceschini A, von Mering C, Jensen LJ, Bork P. STITCH 3: zooming in on protein-chemical interactions. Nucleic Acids Res 2012;40(Database issue):D876-D880

23 Szklarczyk D, Gable AL, Lyon D, et al. STRING v11: protein-protein association networks with increased coverage, supporting functional discovery in genome-wide experimental datasets. Nucleic Acids Res 2019;47(D1):D607-D613

24 Wu C, Liu Y, Yang Y, et al. Analysis of therapeutic targets for SARSCoV-2 and discovery of potential drugs by computational methods. Acta Pharm Sin B 2020;10(05):766-788

$25 \mathrm{Xu} \mathrm{H}$, Zhong L, Deng J, et al. High expression of ACE2 receptor of 2019-nCoV on the epithelial cells of oral mucosa. Int J Oral Sci 2020;12(01):8 
26 O’Boyle NM, Banck M, James CA, Morley C, Vandermeersch T, Hutchison GR. Open Babel: an open chemical toolbox. J Cheminform 2011;3:33

27 Trott O, Olson AJ. AutoDock Vina: improving the speed and accuracy of docking with a new scoring function, efficient optimization, and multithreading. J Comput Chem 2010;31(02):455-461

28 Jin Z, Du X, Xu Y, et al. Structure of $\mathrm{M}^{\text {pro }}$ from SARS-CoV-2 and discovery of its inhibitors. Nature 2020;582(7811):289-293

29 Hoffmann M, Kleine-Weber H, Schroeder S, et al. SARS-CoV-2 cell entry depends on ACE2 and TMPRSS2 and is blocked by a clinically proven protease inhibitor. Cell 2020;181(02):271.e8-280.e8

30 Wang M, Cao R, Zhang L, et al. Remdesivir and chloroquine effectively inhibit the recently emerged novel coronavirus (2019-nCoV) in vitro. Cell Res 2020;30(03):269-271

31 Huang Y, Yang R, Xu Y, Gong P. Clinical characteristics of 36 nonsurvivors with COVID-19 in Wuhan, China. medRxiv 2020; Accessed July 1, 2021 at: https://www.medrxiv.org/content/ 10.1101/2020.02.27.20029009v2

32 Li G, De Clercq E. Therapeutic options for the 2019 novel coronavirus (2019-nCoV). Nat Rev Drug Discov 2020;19(03):149-150

33 Ruan RY, Deng XM, Wang Z, et al. Simultaneous determination of 9 constituents in Pudilan Xiaoyan Oral Liquid by UPLC [in Chinese]. Xibei Yaoxue Zazhi 2021;36:194-197

34 Shao ZB, Zhu YX, Liu SH, Jiang KJ, Liu WW. A review on the clinical application of high frequency traditional Chinese medicine in the treatment of new coronavirus pneumonia. Chin Tradit Herbal Drugs 2020;51:1153-1158

35 Yang A, Liu H, Liu F, et al. Study of the mechanism of the Reyanning mixture involved in treating novel coronavirus pneumonia based on network pharmacology. Nat Prod Commun 2020;15:1-12

36 Li Y, Zhang J, Li S, et al. Efficacy and safety of Reyanning mixture combined with conventional Western medicine for treating COVID-19: a protocol for systematic review and meta-analysis. Medicine (Baltimore) 2021;100(03):e24169

37 Zhai XT, Chen JQ, Jiang $\mathrm{CH}$, et al. Corydalis bungeana Turcz. attenuates LPS-induced inflammatory responses via the suppression of NF-KB signaling pathway in vitro and in vivo. J Ethnopharmacol 2016;194:153-161

38 Lin CW, Tsai FJ, Tsai CH, et al. Anti-SARS coronavirus 3C-like protease effects of Isatis indigotica root and plant-derived phenolic compounds. Antiviral Res 2005;68(01):36-42

39 Fung TS, Liu DX. Activation of the $\mathrm{c}$-Jun $\mathrm{NH}_{2}$-terminal kinase pathway by coronavirus infectious bronchitis virus promotes apoptosis independently of c-Jun. Cell Death Dis 2017;8(12):3215

40 Pleschka S. RNA viruses and the mitogenic Raf/MEK/ERK signal transduction cascade. Biol Chem 2008;389(10):1273-1282

41 Yin Q, Han T, Fang B, et al. K27-linked ubiquitination of BRAF by ITCH engages cytokine response to maintain MEK-ERK signaling. Nat Commun 2019;10(01):1870

42 Mizutani T, Fukushi S, Saijo M, Kurane I, Morikawa S. JNK and PI3k/Akt signaling pathways are required for establishing persistent SARS-CoV infection in Vero E6 cells. Biochim Biophys Acta 2005;1741(1-2):4-10

43 Chen N, Zhou M, Dong X, et al. Epidemiological and clinical characteristics of 99 cases of 2019 novel coronavirus pneumonia in Wuhan, China: a descriptive study. Lancet 2020;395(10223):507-513

44 Schett G, Sticherling M, Neurath MF. COVID-19: risk for cytokine targeting in chronic inflammatory diseases? Nat Rev Immunol 2020;20(05):271-272

45 Bradbury RS, Piedrafita D, Greenhill A, Mahanty S. Will helminth co-infection modulate COVID-19 severity in endemic regions? Nat Rev Immunol 2020;20(06):342

46 Cao X. COVID-19: immunopathology and its implications for therapy. Nat Rev Immunol 2020;20(05):269-270
47 Jackson DJ, Busse WW, Bacharier LB, et al. Association of respiratory allergy, asthma, and expression of the SARS-CoV-2 receptor ACE2. J Allergy Clin Immunol 2020;146(01):203.e3-206.e3

$48 \mathrm{Kai} \mathrm{H}$, Kai M. Interactions of coronaviruses with ACE2, angiotensin II, and RAS inhibitors-lessons from available evidence and insights into COVID-19. Hypertens Res 2020;43(07):648-654

$49 \mathrm{Li} \mathrm{Y}$, Zeng Z, Cao Y, et al. Angiotensin-converting enzyme 2 prevents lipopolysaccharide-induced rat acute lung injury via suppressing the ERK1/2 and NF-KB signaling pathways. Sci Rep 2016;6:27911

50 Fung TS, Liu DX. Human coronavirus: host-pathogen interaction. Annu Rev Microbiol 2019;73:529-557

51 Gao Y, Kang L, Li C, et al. Resveratrol ameliorates diabetes-induced cardiac dysfunction through AT1R-ERK/p38 MAPK signaling pathway. Cardiovasc Toxicol 2016;16(02):130-137

52 Yan YS, Cao X, Zhang Y, et al. Discovery of anti-2019-nCoV agents from 38 Chinese patent drugs toward respiratory diseases via docking screening. Preprints 2020:2020020254. Accessed July 1, 2021 at: https://www.preprints.org/manuscript/202002.0254/v2

53 Chen H, Du Q. Potential natural compounds for preventing 2019nCoV infection. Preprints 2020:2020010358. Accessed July 1, 2021 at: https://www.preprints.org/manuscript/202001.0358/v2

54 Chen F, Chan KH, Jiang Y, et al. In vitro susceptibility of 10 clinical isolates of SARS coronavirus to selected antiviral compounds. J Clin Virol 2004;31(01):69-75

55 Zhou Y, Hou Y, Shen J, et al. Network-based drug repurposing for human coronavirus. medRxiv 2020; Accessed July 1, 2021 at: https://www.medrxiv.org/content/ $10.1101 / 2020.02 .03 .20020263 \mathrm{v} 1$

56 Zheng N, Li Q, Sun S, et al. The synergistic effects of the bupleurum-scutellaria herbal pair in alcoholic liver injury revealed by metabolomics and metallomics. Front Pharmacol 2019;10:254

57 Luan X, Zhang LJ, Li XQ, et al. Compound-based Chinese medicine formula: from discovery to compatibility mechanism. J Ethnopharmacol 2020;254:112687

58 Zhang B, Saatman KE, Chen L. Therapeutic potential of natural compounds from Chinese medicine in acute and subacute phases of ischemic stroke. Neural Regen Res 2020;15(03):416-424

59 Caesar LK, Cech NB. Synergy and antagonism in natural product extracts: when $1+1$ does not equal 2. Nat Prod Rep 2019;36(06): 869-888

60 Shi XQ Yue SJ, Tang YP, et al. A network pharmacology approach to investigate the blood enriching mechanism of Danggui buxue decoction. J Ethnopharmacol 2019;235:227-242

61 Cai FF, Bian YQ, Wu R, et al. Yinchenhao decoction suppresses rat liver fibrosis involved in an apoptosis regulation mechanism based on network pharmacology and transcriptomic analysis. Biomed Pharmacother 2019;114:108863

62 Tao Y, Tian K, Chen J, et al. Network pharmacology-based prediction of the active compounds, potential targets, and signaling pathways involved in Danshiliuhao Granule for treatment of liver fibrosis. Evid Based Complement Alternat Med 2019; 2019:2630357

63 Blaženović I, Kind T, Ji J, Fiehn O. Software tools and approaches for compound identification of LC-MS/MS data in metabolomics. Metabolites 2018;8(02):1-23

64 Schulte-Michels J, Keksel C, Häberlein H, Franken S. Anti-inflammatory effects of ivy leaves dry extract: influence on transcriptional activity of NFKB. Inflammopharmacology 2019;27(02): 339-347

65 Ma Q, Pan W, Li R, et al. Liu Shen capsule shows antiviral and antiinflammatory abilities against novel coronavirus SARS-CoV-2 via suppression of NF-kB signaling pathway. Pharmacol Res 2020; 158:104850 